\title{
Acute abdomen in a patient with rudimentary horn
}

\author{
Najam $\mathbf{R}^{1}$, Gupta $\mathrm{S}^{2}$, Chowdhury $\mathbf{H}^{3}$
}

\begin{abstract}
Unicornuate uterus with a rudimentary horn is a rare condition. These patients present with dysmenorrhoea and chronic pelvic pain and infertility. We present an interesting case of hematometra with non-communicating rudimentary horn with unicornuate uterus. Strong suspicion to this condition helps in early diagnosis and treatment.
\end{abstract}

Key words: rudimentary horn, dysmenorrhoea, hematometra

\section{Introduction}

Unicornuate uterus with non-communicating rudimentary horn is susceptible to many gynaecological and obstetric complications which can occur at any stage of reproductive life $^{1}$. High index of suspicion should be kept in teenagers and young patients presenting with dysmenorrhoea. Unicornuate uterus with a rudimentary horn is a rare type of mullerian duct malformation with a prevalence of 1:10000².

\section{Case report}

A 24 year old nulligravida was admitted to the Department of Obstetrics and Gynaecology, of Teerthanker Mahaveer Medical College, Moradabad, (U.P.), India, with the complaint of severe dysmenorrhoea for past 10-12 years along with primary infertility. She attained menarche at the age 13 and she started having dysmenorrhoea since 14 years. She was married 4 years back and had been on and off treat-ment with GP but was not relieved.

${ }^{1}$ Associate Professor,

${ }^{2}$ Senior Resident,

${ }^{3}$ Professor and Head, Department of Obstetrics and Gynaecology, Teerthanker Mahaveer Medical College and Research Centre, Teerthanker Mahaveer University, Moradabad, (U.P.), India.

Correspondence: R Najam

E-mail: rehananajam01@gmail.com
Vital signs remained stable. On abdominal examination there was tenderness present in the right iliac fossa. Gynaecological examinations revealed a normal vagina and cervix with a bulky uterus. The uterus was deviated to right side and it was tender. She had an approximately $5 \times 4 \mathrm{~cm}$ palpable tender hard mass with restricted mobility in the right adnexal region. Differential diagnosis of chronic endometriosis and chronic ectopic was made. Laboratory values were as follows: haemoglobin $10.5 \mathrm{~g} / \mathrm{dl}$, hematocrit $37 \%$, white blood cell count 12000 per $\mathrm{mm}^{3}$ platelet count 105000 per $\mathrm{mm}^{3}$. Her $\beta$ HCG level was not detectable. CA 125 - was 100 mIU. Ultrasonography revealed a $40 \times 38 \mathrm{~mm}$ smooth, contoured, homogeneous mass with collection in right adnexal region. MRI was done in which a diagnosis of rudimentary horn with hematometra was made. Sonography for kidneys and intravenous pyelography was done which was normal. Decision for laparotomy and excision of rudimentary horn with ipsilateral salpingectomy was taken.

Peroperatively the left round ligament arose from the left uterine cornua however the right round ligament arose from the rudimentary horn. The right sided non communicating functional rudimentary horn of $5 \times 4 \mathrm{~cm}$ in size was attached to the uterus, ovaries were adhered posteriorly and endometrioma of $5 \times 5 \mathrm{~cm}^{2}$ was present on right side, which was adhered to the omentum. Pouch of Douglas was completely obliterated. Endometriotic implants were present on the left side and the left tube was blocked due to fimbrial adhesions. Adhesiolysis and right sided semi hysterectomy with right sided salpingectomy was done (Figure 1 and Figure 2), with removal of endometrioma. Adhesiolysis was done on

\section{Figure 1. Haematometra (Rt horn) with normal Left horn.}

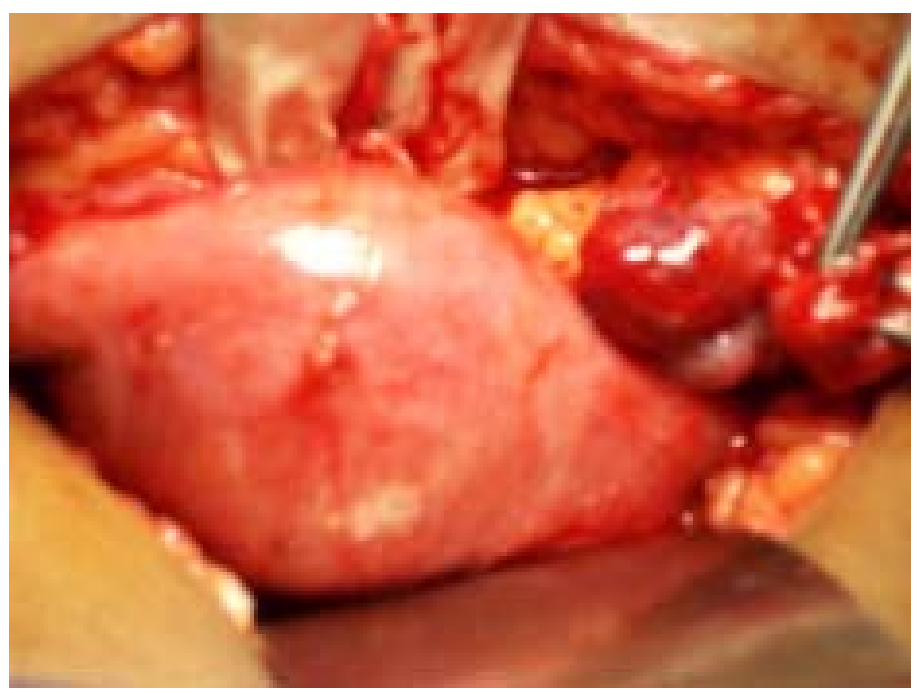


Figure 2, 3. Aspiration of blood from the rudimentary horn

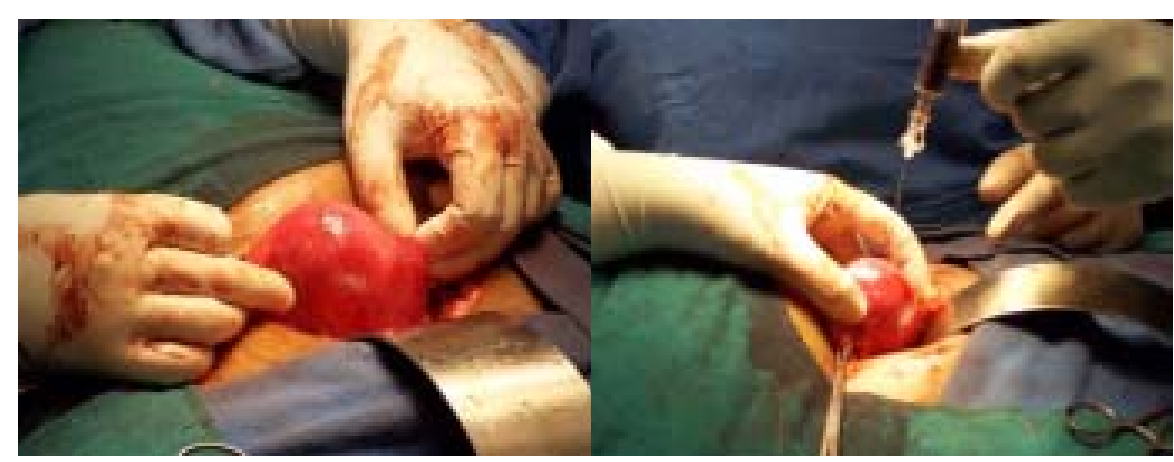

Figure 4. Hemihysterectomy with removal of the rudimentary horn and repair.

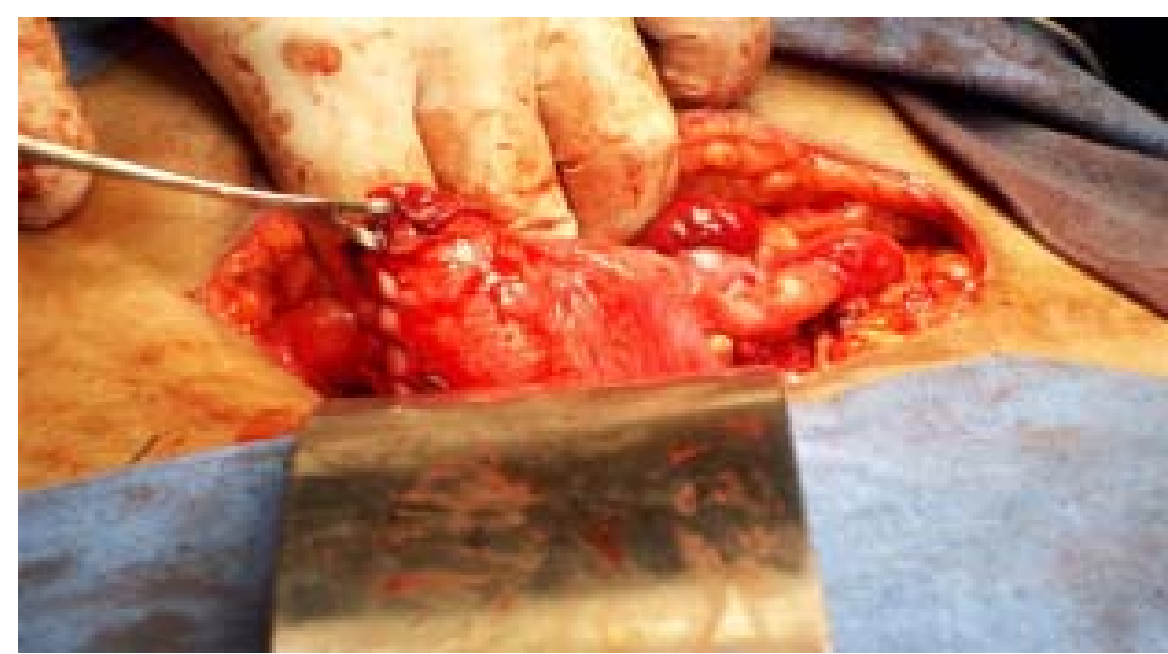

left side also (Figure 3 and Figure 4). After Chromo-tubation the left tube was found to be patent. Postoperative period was uneventful and she was treated with GnRH analogues for prevention of endometriosis.

\section{Discussion}

Unicornuate uterus with a rudimentary horn is the rarest congenital anatomic anomaly of the female genital system². Urinary tract abnormalities are commonly associated with mullerian anomalies. This pathology is classified into 4 groups by the American Society of Reproductive Medicine (ASRM) as Unicornuate uterus with communi-
Diagnosis is missed by inexperienced hands. Three dimensional ultrasound may be used for diagnosis of uterine abnormalities. Laparoscopy is the most accurate diagnostic tool, yet MRI has emerged as a useful and noninvasive tool for diagnosis of uterine anomalies ${ }^{5}$. Once diagnosis is strongly suspected, laparoscopy or laprotomy is a must, excision of the rudimentary horn is advised. Literatures show a low preclinical $(8 \%)$ and preoperative detection rate $(29 \%)$. Evaluation of renal system is advised because of high incidence of urological anomalies. Surgical removal of the noncommunicating horn should be performed once the diagnosis is made especially if it contains functional endometrium, to prevent endometriosis and adverse outcome. In our case, removal of the horn resulted in relief of dysmenorrhoea. The patient is currently undergoing follow up.

\section{REFERENCES}

1. Goel P, Aggarwal A, Devi K, Takkar N, Saha PK, Huria A. Unicornuate uterus with non-communicating rudimentary horn different clinical presentations J. Obstet Gynecol India 2005; 55: 155-8.

2. Rusen Atmaca, Aysegul Tezcan German, feza burak, Ayre Kafkasli acute abdomen in a case with non-communicating rudimentary horn and unicornuate uterus Journal of the Society of Laparoendoscopic Surgeons 2005; 9: 235-7.

ting rudimentary horn, unicorns uterus with non-communicating rudimentary horn, isolated unicornuate uterus and non cavitated unicornuate uterus with non-communicating rudimentary horn ${ }^{3}$. Possible causes of abdominal pain in these patients are the distention of the uterus because of blood accumulation in the noncommunicating cavity of the rudimentary horn, hematometra, pyometra, and torsion ${ }^{4}$. Another cause of abdominal pain in these women is endometriosis ${ }^{5}$. Retrograde menstruation from the ipsilateral tube results in endometriosis supporting retrograde menstruation theor ${ }^{5}$. Sensitivity of USG is only $26 \%$.
3. The American Fertility Society. The American Fertility Society Classifications of Adnexal adhesions, distal tubal occlusion, secondary to tubal ligation, tubal pregnancies, mullerian anomilies and intra uterine adhesions. Fertil Steril 1988; 48: 944-55.

4. Kuscu NK, Lasin S, Kartal O. Rupture of rudimentary horn pregnancy at the 15th of gestation: a case report. Eurobstet Gynecol Reprod Biol. 2002; 102: 209-10.

5. Matalliotakis IM, Kourmntakis G, Kourmantakis EE. Pulmonary endometriosis in a patient with unicornuate uterus and non communicating rudimentary horn. Fertil steril. 2002; 78: 183-5. 